\title{
Stimulation of phosphatidylethanolamine synthesis in isolated rat hepatocytes by phorbol 12-myristate 13-acetate
}

\author{
Lilian B.M. Tijburg, Martin Houweling, Math J.H. Geelen \\ and Lambert M.G. van Golde \\ Laboratory of Veterinary Biochemistry, University of Utrecht, Utrecht (The Netherlands)
}

(Received 15 June 1987)

Key words: Phosphatidylethanolamine; Phospholipid synthesis; Phorbol ester; (Rat hepatocyte)

Incubation of freshly isolated rat hepatocytes in the presence of phorbol 12-myristate 13-acetate stimulates the incorporation of $\left[1,2-{ }^{14} \mathrm{C}\right]$ ethanolamine into phosphatidylethanolamines. This stimulation is strongly dependent on the ethanolamine concentration in the medium and becomes apparent at ethanolamine concentrations above $25 \mu \mathrm{M}$. Treatment of hepatocytes with phorbol 12-myristate 13-acetate results in a decreased labelling of intracellular ethanolamine, ethanolaminephosphate and CDPethanolamine. Exposure of cells to phorbol 12-myristate 13-acetate induces an increase of the activity of the enzymes CTP: ethanolaminephosphate cytidylyltransferase and ethanolaminephosphotransferase. These effects are accompanied by a decrease of the pool size of ethanolaminephosphate and CDPethanolamine and an increase of the level of diacylglycerols after $30 \mathrm{~min}$ of incubation in the presence of phorbol 12-myristate 13-acetate. Upon prolonged incubation, the CDPethanolamine and diacylglycerol pools are restored to the level found in untreated cells. These results indicate that stimulation of phosphatidylethanolamine synthesis by phorbol 12-myristate 13-acetate is probably exerted at the level of CTP: ethanolaminephosphate cytidylyltransferase, although there may be an additional effect on the subsequent step of phosphatidylethanolamine synthesis, the formation of phosphatidylethanolamines from CDPethanolamine and diacylglycerols.

\section{Introduction}

Isolated cells incubated in the presence of tumor promoters undergo a wide range of alterations [1]. One of the early metabolic changes observed, is an enhancement of phosphatidylcholine synthesis. Phorbol 12-myristate 13-acetate (PMA), a potent tumor-promoting phorbol ester, has been shown

\footnotetext{
Abbreviations: HPLC, high-performance liquid chromatography; PMA, phorbol 12-myristate 13-acetate.

Correspondence: L.B.M. Tijburg, Laboratory of Veterinary Biochemistry, P.O. Box 80.177, 3508 TD Utrecht, The Netherlands.
}

to stimulate the incorporation of labelled choline into phosphatidylcholines in several cell types [2-7]. Paddon and Vance [5] suggested that this effect might be due to an activation of CTP: cholinephosphate cytidylyltransferase (EC 2.7.7.15), the regulatory enzyme in the synthesis de novo of phosphatidylcholines [8]. Recently, it was indeed demonstrated that PMA can enhance the activity of this enzyme in HeLa cells [9] as well as in myoblasts [10].

Stimulation of phosphatidylcholine synthesis by tumor-promoting phorbol esters can be correlated with their biological activity as comitogen $[2,3]$. As phosphatidylcholine is only one of the phospholipids occurring in membranes, it is not unlikely to 
assume that the increased membrane biogenesis is accompanied by an enhancement of the synthesis of other membrane phospholipids as well. In this paper, we report the effects of PMA on the synthesis of phosphatidylethanolamines. The data presented here demonstrate that tumor-promoting phorbol esters enhance the incorporation of [1,2-

${ }^{14} \mathrm{C}$ ]ethanolamine into phosphatidylethanolamines by isolated rat hepatocytes. The observed activation of CTP : ethanolaminephosphate cytidylyltransferase in combination with an enhancement of the activity of ethanolaminephosphotransferase is in line with an increased synthesis of this phospholipid.

\section{Materials and Methods}

Materials. $\left[1,2-{ }^{14} \mathrm{C}\right]$ Ethanolamine was obtained from New England Nuclear (Dreieichenhain, F.R.G.). $\quad \mathrm{CDP}\left[1,2-{ }^{14} \mathrm{C}\right]$ ethanolamine was purchased from ICN Radiochemicals (Irvine, U.K.) and $\left[1,2-{ }^{14} \mathrm{C}\right]$ ethanolaminephosphate was prepared according to Sundler [11]. PMA, $4 \alpha$-phorbol-12,13-didecanoate, $4 \beta$-phorbol-12,13-didecanoate, bovine scrum albumin (fraction V), collagenase (type I), glycerol kinase and glycerol-3phosphate dehydrogenase were purchased from Sigma (St. Louis, MO, U.S.A.) and dansyl chloride was from Janssen Chimica (Beerse, Belgium). All other chemicals were of analytical grade and obtained from Baker Chemical Co. (Deventer, The Netherlands).

Isolation and incubation of hepatocytes. Freshly isolated hepatocytes were obtained from male Wistar rats $(200-250 \mathrm{~g})$, which were meal-fed a stock pelleted diet and had free access to water. Hepatocytes were isolated essentially according to Seglen [12] as described earlier [13]. The isolated hepatocytes were suspended in Krebs-Ringer bicarbonate buffer ( $\mathrm{pH}$ 7.4) supplemented with 10 $\mathrm{mM}$ glucose, $1 \%$ defatted and dialyzed bovine serum albumin, $0.1 \mathrm{mM}$ choline and $0.1 \mathrm{mM}$ ethanolamine. Incubations were carried out in 25 ml-Erlenmeyer flasks, which were continuously gassed with $95 \%$ oxygen $/ 5 \%$ carbon dioxide, and contained 4-5 mg cellular protein $/ \mathrm{ml}$. Phorbol esters $\left(10^{-6} \mathrm{M}\right)$ were added to the incubations dissolved in dimethylsulfoxide. The final dimethylsulfoxide concentration was $0.1 \%$ in phorbol es- ter-containing as well as in control incubations.

Isolation of phospholipids and of water-soluble precursors of phosphatidylethanolamines. Incubations were terminated after the indicated periods by the addition of 6.5 vols. chloroform/methanol $(1: 1 \mathrm{v} / \mathrm{v})$. Lipids were extracted according to Sundler et al. [14]. Phospholipids were subsequently separated by thin-layer chromatography on silica gel $\mathrm{G}$ using chloroform/methanol/water $(65: 35: 4 \mathrm{v} / \mathrm{v})$ as developing solvent. Neutral lipids were also separated on silica gel $G$ with petroleum ether (b.p. $40-60^{\circ} \mathrm{C}$ )/ether/acetic acid $(80: 2: 2 \mathrm{v} / \mathrm{v})$ as the eluent.

Extraction of water-soluble metabolites was carried out exactly as described before [15]. Radiolabelled water-soluble phosphatidylethanolamine precursors were separated by thin-layer chromatography on silica gel $\mathrm{H}$ with $0.5 \% \mathrm{NaCl} /$ methanol/ $\mathrm{NH}_{4} \mathrm{OH}(50: 50: 5 \mathrm{v} / \mathrm{v})$ as the mobile phase [16]. Spots of ethanolamine intermediates were detected with ninhydrin. Pool sizes of phosphatidylethanolamine precursors were determined according to a novel method which will be described in detail elsewhere (Tijburg, L.B.M., Houweling, M., Geelen, M.J.II. and van Golde, L.M.G., unpublished data). Briefly, the watersoluble metabolites were extracted as described above and separated by HPLC on an LKB TSK DEAE-3W column $(7.5 \times 150 \mathrm{~mm})$, which was eluted with $0.01 \mathrm{M}$ sodium phosphate buffer $(\mathrm{pH}$ 5.6). Free ethanolamine and ethanolamine obtained from ethanolaminephosphate after hydrolysis in $6 \mathrm{M} \mathrm{HCl}$ for $18 \mathrm{~h}$ at $110^{\circ} \mathrm{C}$ were determined fluorimetrically as dansyl derivatives according to Sundler and Åkesson [17]. CDPethanolamine-containing fractions were combined and completely resolved from CDPcholine by HPLC on an LKB Lichrosorb reversed-phase $\mathrm{C} 18$ column $(4 \times 250$ $\mathrm{mm}$ ) with $0.1 \%$ sodium phosphate buffer $(\mathrm{pH} 2.5)$ as mobile phase. CDPethanolamine was quantified spectrophotometrically at $280 \mathrm{~nm}$.

Measurement of enzyme activities. At the end of the incubation period the cells were washed twice by centrifugation and resuspension and homogenized with 50 strokes of a tight-fitting Dounce homogenizer in ice-cold homogenization buffer containing $0.145 \mathrm{M} \mathrm{NaCl} / 10 \mathrm{mM}$ Tris- $\mathrm{HCl} / 1$ $\mathrm{mM}$ EDTA $/ 10 \mathrm{mM} \mathrm{NaF}$ [18]. Subcellular fractionation was performed exactly as described in 
Ref. 15. Ethanolamine kinase was determined in the cytosolic fraction as described by Weinhold and Rethy [19]. CTP : ethanolaminephosphate cytidylyltransferase was determined essentially according to Sundler [11] modified as described in Ref. 15. Ethanolaminephosphotransferase was determined with endogenous diacylglycerols exactly as described previously [15].

Other methods. After separation of the neutral lipids as described above, diacylglycerols were extracted from the silica, hydrolyzed and determined essentially according to Wieland [20] modified as described previously [15]. Protein was determined according to Lowry et al. [21] using bovine serum albumin as a standard.

Results are presented as means \pm S.D. of the number of experiments indicated. Where two experiments were carried out results are presented as means \pm ranges. Significance was determined by the Student's $t$-test.

\section{Results}

Effects of various phorbol esters on phosphatidylethanolamine synthesis in pulse-label experiments

Incubations of hepatocytes in the presence of PMA resulted in an increased incorporation of $\left[1,2-{ }^{14} \mathrm{C}\right]$ ethanolamine into phosphatidylethanolamines. This stimulation was maximal at a concentration of $10^{-6} \mathrm{M}$ PMA. At this concentration, the incorporation of labelled ethanolamine into phosphatidylethanolamines in PMA-treated cells was $143 \%$ of the incorporation in control incubations (Table I). Half-maximal stimulation was reached at $5 \cdot 10^{-8} \mathrm{M}$ PMA (results not shown). Table I demonstrates that PMA did not only stimulate the incorporation of labelled ethanolamine into phosphatidylethanolamines, but also that into phosphatidylcholines. Another potent tumor-promoter, $4 \beta$-phorbol-12,13-didecanoate also enhanced phosphatidylethanolamine synthesis, whereas its biologically inactive stereoisomer, $4 \alpha$-phorbol-12,13-didecanoate did not affect the incorporation of labelled ethanolamine into phosphatidylethanolamines (Table I).

The stimulating effect of PMA on phosphatidylethanolamine synthesis was strongly dependent on the ethanolamine concentration in the medium (Fig. 1). In agreement with the results of
TABLE I

EFFECTS OF PHORBOL ESTERS ON THE INCORPORATION OF $\left[1,2-{ }^{14} \mathrm{C}\right] E$ THANOLAMINE INTO PHOSPHATIDYLETHANOLAMINES AND PHOSPHATIDYLCHOLINES

Hepatocytes were incubated for $60 \mathrm{~min}$ in the absence or presence of the indicated phorbol esters $\left(10^{-6} \mathrm{M}\right)$. Incubations were terminated and phospholipids were extracted as described in Materials and Methods. Values are means \pm S.D. with the number of experiments in parentheses. In the case of $4 \beta$ phorbol-12,13-didecanoate values are expressed as means \pm ranges.

\begin{tabular}{|c|c|c|}
\hline & \multicolumn{2}{|c|}{$\begin{array}{l}{\left[1,2-{ }^{14} \mathrm{C}\right] \text { Ethanolamine incorporated }} \\
(\mathrm{nmol} / \mathrm{mg} \text { protein per } \mathrm{h})\end{array}$} \\
\hline & $\begin{array}{l}\text { phosphatidyl- } \\
\text { ethanolamines }\end{array}$ & $\begin{array}{l}\text { phosphatidyl- } \\
\text { cholines }\end{array}$ \\
\hline Control & $4.18 \pm 0.59(10)$ & $0.38 \pm 0.15(10)$ \\
\hline PMA & $5.97 \pm 0.79(10)^{a}$ & $0.57 \pm 0.20(10)^{b}$ \\
\hline $\begin{array}{l}4 \beta \text {-Phorbol-12,13- } \\
\text { didecanoate }\end{array}$ & $6.70 \pm 0.16$ & $0.59 \pm 0.08$ \\
\hline $\begin{array}{c}4 \alpha \text {-Phorbol-12,13- } \\
\text { didecanoate }\end{array}$ & $4.34 \pm 0.99$ & $0.41 \pm 0.08$ \\
\hline
\end{tabular}

a Significantly different from control, $P<0.01$.

b Significantly different from control, $P<0.05$.

Sundler and Åkesson [17], maximal incorporation of ethanolamine into phosphatidylethanolamines was reached at concentrations between 50 and 100

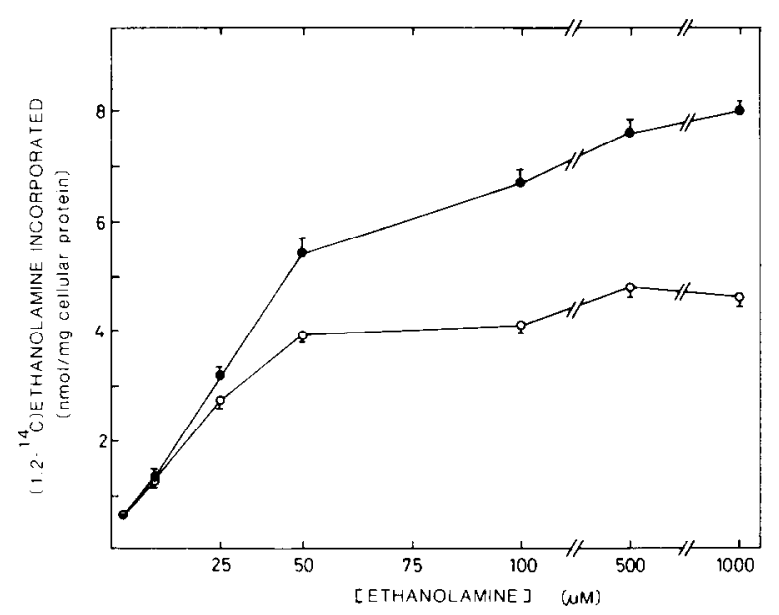

Fig. 1. The effect of the ethanolamine concentration on the incorporation of $\left[1,2-{ }^{14} \mathrm{C}\right]$ ethanolamine into phosphatidylethanolamines. Freshly isolated rat hepatocytes were incubated for $60 \mathrm{~min}$ in the absence $(O)$ or presence $(\bullet)$ of PMA $\left(10^{-6}\right.$ $M)$. Values are the means \pm S.D. of triplicate incubations of one representative experiment which was repeated once with similar results. 
$\mu \mathrm{M}$, whereas the maximal stimulating effect of PMA was reached at a concentration above 100 $\mu \mathrm{M}$. At ethanolamine concentrations below 25 $\mu \mathrm{M}$, the PMA effect on phosphatidylethanolamine synthesis was marginal. The incorporation of labelled ethanolamine into phosphatidylcholines was also dependent on the ethanolamine concentration and was maximal at concentrations between 50 and $100 \mu \mathrm{M}$ (not shown).

To exclude the possibility that the stimulating effect of PMA on phosphatidylethanolamine synthesis was a result of an enhanced uptake of ethanolamine, we determined the incorporation of labelled ethanolamine into water-soluble phosphatidylethanolamine precursors in the presence or absence of PMA. Incubation of hepatocytes in the presence of PMA resulted within $10 \mathrm{~min}$ in a stimulation of phosphatidylethanolamine synthesis (Fig. 2, panel D). On exposure of hepatocytes to PMA the incorporation of ethanolamine into water-soluble metabolites was strongly inhibited (Fig. 2). Treatment of hepatocytes with PMA for less than $40 \mathrm{~min}$ resulted in a decreased amount of labelled intracellular ethanolamine (Fig. 2, panel A). The amount of label recovered in ethanolaminephosphate after $40 \mathrm{~min}$ of incubation in the presence of PMA, declined to $65 \%$ of the value found in control incubations (Fig. 2, panel B), whereas the amount of labelled CDPethanolamine declined to $77 \%$ of that in untreated cells (Fig. 2, panel C). After an incubation period of $60 \mathrm{~min}$ in the presence of PMA, the amount of label recovered in CDPethanolamine was no longer significantly different from that in control hepatocytes.

Effects of PMA on the activities of the enzymes of the CDPethanolamine pathway

In order to investigate the mechanism of stimulation of phosphatidylethanolamine synthesis by tumor-promoting phorbol esters, we decided to determine the activities of the enzymes involved in the CDPethanolamine pathway. Table II demonstrates that PMA did not affect the activity of ethanolamine kinase. However, the activity of ethanolaminephosphate cytidylyltransferase was indeed enhanced in PMA-treated hepatocytes compared to control cells (Table II). This might indicate that the effect of PMA on phosphatidyl-
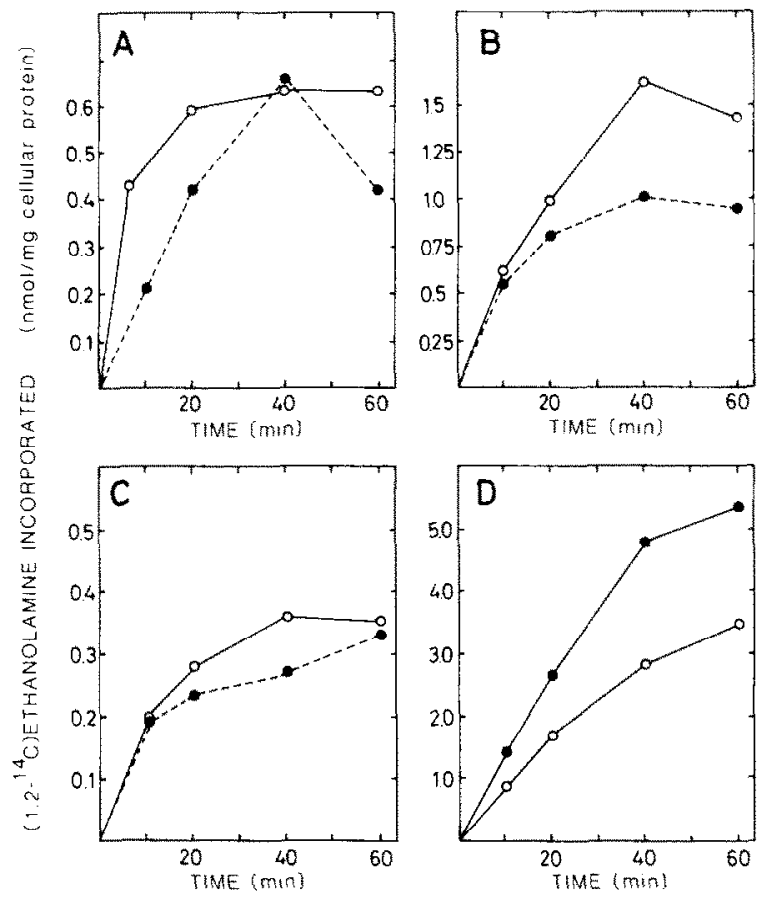

Fig. 2. The effect of PMA on the incorporation of 11.2${ }^{14}$ Clethanolamine into phosphatidylethanolamines and their water-soluble precursors. Hepatocytes were incubated with $\left[1,2{ }^{14} \mathrm{C}\right]$ ethanolamine in the absence $(0)$ or presence $(6)$ of $10^{-6} \mathrm{M}$ PMA. At various times up to $1 \mathrm{~h}$, cells were washed and the radioactivity incorporated into cellular ethanolamine metabolites was determined. Values are the means of duplicate incubations of one representative experiment which was repeated once with similar results. A, ethanolamine; $B_{n}$ ethanolaminephosphate; C, CDPethanolamine; D, phosphatidylethanolamines.

ethanolamine synthesis was exerted at the level of the conversion of ethanolaminephosphate to CDPethanolamine, the putative control point of the pathway [17]. However, the activity of ethanolaminephosphotransferase was also enhanced in hepatocytes incubated in the presence of PMA $\left(10^{-6} \mathrm{M}\right)$ compared to control cells (Table II). The latter effect might be the result of the enhancement of the amount of cellular diacylglycerols in the presence of PMA. Fig. 3 demonstrates that PMA induced a fast increase of the amount of diacylglycerols. After $10 \mathrm{~min}$ of incubation in the presence of PMA the amount of diacylglycerols was $156 \%$ of that in untreated cells. Upon prolonged treatment with PMA the amount of diacylglycerols was restored to the level found in control cells (Fig. 3). 


\section{TABLE II}

EFFECTS OF PMA ON THE ACTIVITIES OF THE ENZYMES OF THE CDP-ETHANOLAMINE PATHWAY

Hepatocytes were incubated for $60 \mathrm{~min}$ in the absence or presence of PMA $\left(10^{-6} \mathrm{M}\right)$. Subcellular fractionation and subsequent determination of the enzyme activities were carried out as described in Materials and Methods. Values are expressed as means \pm S.D. of the number of experiments indicated in parentheses.

\begin{tabular}{lll}
\hline Enzyme & $\begin{array}{l}\text { Specific activity } \\
\text { control (nmol/mg } \\
\text { protein per min) }\end{array}$ & $\begin{array}{l}\text { PMA } \\
\% \text { of control }\end{array}$ \\
\hline $\begin{array}{l}\text { Ethanolamine kinase } \\
\begin{array}{l}\text { Ethanolaminephosphate } \\
\text { cytidylyltransferase }\end{array}\end{array}$ & $2.12 \pm 0.51(5)$ & $116.8 \pm 4.4^{\mathrm{a}}$ \\
$\begin{array}{l}\text { Ethanolamine- } \\
\text { phosphotransferase }\end{array}$ & $0.10 \pm 0.03(4)$ & $133.0 \pm 16.7^{\mathrm{b}}$ \\
\hline
\end{tabular}

a Significantly different from control, $P<0.01$.

${ }^{b}$ Significantly different from control, $P<0.05$.

Effects of PMA on the pool sizes of water-soluble precursors of phosphatidylethanolamines

The results of the effects of PMA on the en-

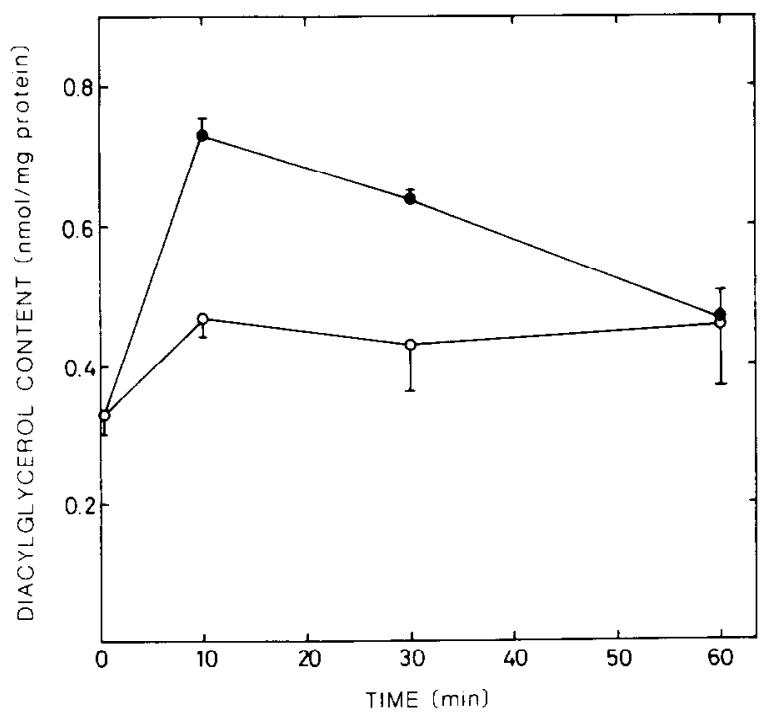

Fig. 3. The effect of PMA on the amount of diacylglycerols in hepatocytes incubated in the absence $(O)$ or presence $(\bullet)$ of $10^{-6}$ M PMA. At various times up to $1 \mathrm{~h}$, the incubations were terminated and diacylglyccrols wcre cxtracted and determined as described in Materials and Methods. Values are means \pm ranges of duplicate incubations of one representative cell preparation. The experiment was repeated with comparable results. zyme activities, i.e., an increase of the activity of ethanolaminephosphate cytidylyltransferase as well as ethanolaminephosphotransferase (Table II), are strongly supported by the results of the determination of the pool sizes of water-soluble phosphatidylethanolamine precursors (Table III). Treatment with PMA did not affect the amount of cellular ethanolamine. However, incubation in the presence of PMA for 30 as well as 60 min resulted in a significant decrease of the amount of ethanolaminephosphate (Table III). This indicates that the conversion of ethanolaminephosphate to cDPethanolamine was indeed enhanced. PMA also diminished the CDPethanolamine pool size after 30 min of incubation with PMA. However, this level was restored upon prolonged exposure to PMA.

\section{Discussion}

Although it has been known for years that tumor-promoting phorbol esters stimulate the incorporation of labelled choline into phosphatidylcholines in a wide variety of cell types [2-7], the effects of these compounds on the synthesis of other phospholipids has not yet been clarified. In this paper we demonstrate that tumor-promoting

\section{TABLE III}

EFFECTS OF PMA ON THE POOL SIZES OF WATERSOLUBLE PHOSPHATIDYLETHANOLAMINE PRECURSORS

Hepatocytes were incubated in triplicate for 30 or $60 \mathrm{~min}$ in the absence of presence of PMA. Determination of water-soluble ethanolamine intermediates was carried out as described in Materials and Methods. Results are given as means \pm ranges of two different cell preparations relative to the ethanolaminecontaining compounds in control incubations. The absolute values of the pool sizes in control incubations (expressed in $\mathrm{nmol} / \mathrm{mg}$ cellular protein) were at $30 \mathrm{~min}$ : ethanolamine, $1.61 \pm 0.08$; ethanolaminephosphate, $7.35 \pm 0.01$; CDPethanolamine, $0.291 \pm 0.019$; and at $60 \mathrm{~min}$ : ethanolamine, $1.34 \pm$ 0.07 ; ethanolaminephosphate, $6.2 \pm 1.8 ;$ CDPethanolamine, $0.245 \pm 0.015$

\begin{tabular}{llc}
\hline & \multicolumn{2}{l}{ Pool size (\% of control) } \\
\cline { 2 - 3 } & $30 \mathrm{~min}$ & $60 \mathrm{~min}$ \\
\hline Ethanolamine & $90 \pm 5$ & $100 \pm 22$ \\
Ethanolaminephosphate & $56 \pm 6$ & $79.6 \pm 2.6$ \\
CDPethanolamine & $78 \pm 4$ & $110.5 \pm 2.5$ \\
\hline
\end{tabular}


phorbol esters stimulate the synthesis of phosphatidylethanolamines from labelled ethanolamine by freshly isolated rat hepatocytes. Our results with labelled ethanolamine are in line with observations of other investigators in other cell types $[10,22]$ who demonstrated that PMA enhanced the incorporation of ${ }^{32} \mathrm{P}$ into phosphatidylethanolamines. The differences between our study and that of Liscovitch et al. [7], who reported that the incorporation of labelled ethanolamine was not enhanced in PMA-treated NG 108-15 cells, might be due to the low ethanolamine concentration used by these investigators. Fig. 1 clearly demonstrates that it is only at ethanolamine concentrations above $25 \mu \mathrm{M}$ that the stimulating effect of PMA on phosphatidylethanolamine synthesis becomes apparent. Incorporation of labelled ethanolamine into phosphatidylcholines, i.e., $N$-methylation of phosphatidylethanolamines, is also enhanced in the presence of PMA (Table I). However, from the results presented in this paper it can not be conclusively determined whether the latter effect is due to an enhanced specific activity of phosphatidylethanolamines or to an activation of phosphatidylethanolamine- $N$-methyltransferase. Fig. 2 (panel D) demonstrates that the enhancement of the formation of phosphatidylethanolamines by PMA is a very fast effect. Incubation of hepatocytes in the presence of PMA results within 10 $\min$ in an increased incorporation of [1,2${ }^{14}$ C]ethanolamine into phosphatidylethanolamines. In this respect there might be a clear distinction between the stimulating effect of PMA on the synthesis of phosphatidylcholines and that of phosphatidylethanolamines. Several investigators demonstrated that the PMA-stimulated incorporation of choline into phosphatidylcholines shows a lag time in various cell types $[2,4,7]$. It has been suggested $[4,6,23]$ that this stimulation is preceded by, and might possibly be a result of, a phospholipase C-mediated hydrolysis of phosphatidylcholines. Our observations indicate (Fig. 2) that the stimulation of phosphatidylethanolamine synthesis is an acute effect which is not caused by an enhanced phospholipase $\mathrm{C}$-mediated hydrolysis of phosphatidylethanolamines.

Exposure of hepatocytes to PMA induced a considerable decrease of the amount of labelled ethanolamine metabolites (Fig. 2, panels A-C). This effect might be due either to an accelerated flow of ethanolamine through the pathway as a result of an enhanced synthesis, or to an enhanced release of ethanolamine intermediates into the medium. The latter phenomenon was described by several investigators for choline in various cell types $[4,24,25]$ and recently also for ethanolamine in NG 108-15 cells [25]. However, in pulse-chase experiments with labelled ethanolamine, we could not detect an increase of the release of labelled ethanolamine nor of ethanolaminephosphate into the medium in the presence of PMA (Tijburg, L.B.M., unpublished observations). Therefore, it is unlikely that the PMA-induced decrease of the amount of labelled water-soluble phosphatidylethanolamine precursors (Fig. 2) is due to a stimulation of the release of water-soluble ethanolamine metabolites.

The results of the determination of the activities of the enzymes involved in the synthesis de novo of phosphatidylethanolamines (Table II) support the idea that PMA stimulates the formation of this phospholipid. Incubation of hepatocytcs in the presence of PMA induced a small, but significant increase of the activity of ethanolaminephosphate cytidylyltransferase (Table II). Although this effect was rather small, it was strongly corroborated by the decline of the pool size of ethanolaminephosphate in the presence of PMA (Table III). The nature of the PMAinduced activation of ethanolaminephosphate cytidylyltransferase is, as yet, unclear. Cook and Vance [26] investigated the mechanism whereby phorbol esters activate cholinephosphate cytidylyltransferase. Their study indicates that this activation is neither a result of an intracellular increase of fatty acyl chains, which might induce translocation of the enzyme, nor of a direct phosphorylation of cholinephosphate cytidylyltransferase by protein kinase $C$. The mechanism whereby tumor-promoting phorbol esters stimulate ethanolaminephosphate cytidylyltransferase remains to be investigated. The increased activity of ethanolaminephosphotransferase might be attributed to a transient increase of the amount of diacylglycerols (Fig. 3). It is generally assumed [27] that PM $\Lambda$ exerts its biological effect by mimicking the action of diacylglycerols in activat- 
ing protein kinase $\mathrm{C}$. However, we also found, in line with observations in hepatocytes [28] as well as in other cell types $[6,22]$, that PMA itself enhanced the cellular amount of diacylglycerols. This effect might be due to an enhanced phospholipase C-mediated hydrolysis of phosphatidylcholines $[6,22]$ or to an increased synthesis of fatty acids [29], although an inhibition of diacylglycerol lipase [30] can not be excluded.

Recently, we demonstrated that vasopressin exerted a stimulating effect on the incorporation of labelled ethanolamine into phosphatidylethanolamines [15]. Pulse-chase experiments with labelled ethanolamine as well as determination of the pool size of ethanolaminephosphate indicated that the conversion of ethanolaminephosphate to CDPethanolamine was enhanced. However, we were not able to detect any change in the activity of ethanolaminephosphate cytidylyltransferase. In this paper, we demonstrate that a stimulation of phosphatidylethanolamine synthesis by PMA was, indeed, accompanied by an activation of the cytidylyltransferase. Besides, vasopressin as well as PMA increased the activity of ethanolaminephosphotransferase. It may be speculated that the velocity of phosphatidylethanolamine synthesis is not only regulated by the activity of ethanolaminephosphate cytidylyltransferase, but that the phosphotransferase activity, which is highly dependent on the amount of available diacylglycerols, plays a role, the importance of which is, as yet, unknown.

\section{acknowledgements}

These investigations were supported by the Netherlands Foundation for Chemical Research (SON) with financial aid from the Netherlands Organization for the Advancement of Pure Research (ZWO).

\section{References}

1 Weinstein, I.B. (1981) J. Supramol. Struct. Cell Biochem. $17,99-120$

2 Wert?, P.W. and Mueller, G.C. (1978) Cancer Res, 38 , $2900-2904$
3 Kinzel, V., Kreibrich, G., Hecker, E. and Süss, R. (1979) Cancer Res. 39, 2743-2752

4 Guy, G.R. and Murray, A.W. (1982) Cancer Res. 42, 1980-1985

5 Paddon, H.B. and Vance, D.E. (1980) Biochim. Biophys. Acta $620,636-640$

6 Grove, R.I. and Schimmel, S.D. (1982) Biochim. Biophys. Acta $711,272-280$

7 Liscovitch, M., Freese, A., Blusztajn, J.K. and Wurtman, R.J. (1986) J. Neurochem. 47, 1936-1941

8 Pelech, S.L. and Vance, D.E. (1984) Biochim. Biophys. Acta 779, 217-251

9 Pelech, S.L., Paddon, H.B. and Vance, D.E. (1984) Biochim. Biophys. Acta 795, 447-451

10 Hill, S.A., McMurray, W.C. and Sanwal, B.D. (1984) Can. J. Biochem. Cell Biol. 62, 369-374

11 Sundler, R. (1975) J. Biol. Chem. 250, 8585-8590

12 Seglen, P.O. (1976) Methods Cell Biol. 13, 29-83

13 Haagsman, H.P., Van den Heuvel, J.M., Van Golde, L.M.G. and Geelen, M.J.H. (1984) J. Biol. Chem. 259, 11273-11278

14 Sundler, R. Ảkesson, B. and Nilsson, A. (1974) J. Biol. Chem. 249, 5102-5107

15 Tijburg, L.B.M., Schuurmans, E.A.J.M., Geelen, M.J.H. and Van Golde, L.M.G. (1987) Biochim. Biophys. Acta 919, 49-57

16 Pritchard, P.H. and Vance, D.E. (1981) Biochem. J. 196, 261-267

17 Sundler, R. and Åkesson, B, (1975) J. Biol. Chem. 250, 3359-3367

18 Pelech, S.L., Pritchard, P.H., Brindley, D.N. and Vance, D.E. (1983) J. Biol. Chem. 258, 6782-6788

19 Weinhold, P.A. and Rethy, V.B. (1974) Biochemistry 13 , $5135-5145$

20 Wieland, O. (1970) in Methoden der enzymatischen Analyse (Bergmeyer, H.U., ed.), 2nd Fdn., pp. 1367-1372, Verlag Chemie, Weinheim

21 Lowry, O.H., Rosebrough, N.J., Farr, A.L. and Randall, R.J. (1951) J. Biol. Chem. 193, 265-275

22 Takuwa, N., Takuwa, Y. and Rasmussen, H. (1987) Biochem. J. 243, 647-653

23 Daniel, L.W., Waite, M. and Wykle, R.L. (1986) J. Biol. Chem. 261, 9128-9132

24 Mufson, R.A., Okin, E and Weinstein, I.B. (1981) Carcinogenesis 2, 1095-1102

25 Liscovitch, M., Blusztajn, J.K., Freese, A. and Wurtman, R.J. (1987) Biochem. J. 241, 81-86

26 Cook, H.W. and Vance, D.E. (1985) Can. J. Biochem. Cell Biol. 63, 145-151

27 Nishizuka, Y. (1986) Science 233, 305-312

28 Bocckino, S.B., Blackmore, P.F. and Exton, J.H. (1985) J. Biol. Chem. 260, 14201-14207

29 Vaartjes, W.I. and De Haas, C.G.M. (1985) Biochem. Biophys. Res, Commun. 129, 721-726

30 Chabbot, H. and Cabot, M.C. (1986) Proc. Natl. Acad. Sci. USA $83,3126-3130$ 